\title{
Identification of Persons Who Are Responsive To Wood Smoke Particle-Induced Airway Inflammation With Assessment of The Effect of GSTM1, Asthma Status And Sex On This Response.
}

David B. Peden ( $\sim$ david_peden@med.unc.edu )

University of North Carolina at Chapel Hill https://orcid.org/0000-0003-4526-4627

\section{Laura Zhou}

University of North Carolina at Chapel Hill

Allison J. Burbank

University of North Carolina at Chapel Hill

Martha Almond

University of North Carolina at Chapel Hill

Michelle L. Hernandez

University of North Carolina at Chapel Hill

Katherine H Mills

University of North Carolina at Chapel Hill

Terry L. Noah

University of North Carolina at Chapel Hill

Heather Wells

University of North Carolina at Chapel Hill

Haibo Zhou

University of North Carolina at Chapel Hill

Neil E. Alexis

University of North Carolina at Chapel Hill

Research

Keywords: Wood smoke particle exposure, airway neutrophil response, responder status

Posted Date: November 17th, 2021

DOI: https://doi.org/10.21203/rs.3.rs-1065589/v1

License: (9) This work is licensed under a Creative Commons Attribution 4.0 International License.

Read Full License 

1 Identification of Persons Who Are Responsive to Wood Smoke Particle-induced Airway

2 Inflammation with assessment of the effect of GSTM1, asthma status and sex on this 3 response.

4

5 David B. Peden, MD ${ }^{1,2 *}$, Laura Zhou, MS ${ }^{1,4 *}$, Allison J. Burbank, MD ${ }^{2,4 *}$, Martha Almond, RRT ${ }^{1}$,

6 Michelle L. Hernandez, MD ${ }^{2,4}$, Katherine H Mills, MS², Terry L. Noah, MD ${ }^{1,3}$, Heather Wells,

$7 \mathrm{MS}^{1}$, Haibo Zhou, $\mathrm{PhD}^{1,4}$ and Neil E. Alexis, $\mathrm{PhD}^{1,2}$. ( ${ }^{*} \mathrm{DBP}, \mathrm{AB}$ and $\mathrm{LZ}$ contributed equally to 8 this work)

10 1. Center for Environmental Medicine, Asthma and Lung Biology, School of Medicine, 11 University of North Carolina at Chapel Hill, Chapel Hill, NC

12 2. Division of Allergy \& Immunology, Department of Pediatrics, School of Medicine,

13 University of North Carolina at Chapel Hill, Chapel Hill, NC

14 3. Division of Pulmonology, Department of Pediatrics, School of Medicine, University of 15 North Carolina at Chapel Hill, Chapel Hill, NC

16 4. Childrens Research Institute, Department of Pediatrics, School of Medicine, University of $17 \quad$ North Carolina at Chapel Hill, Chapel Hill, NC

18 5. Department of Biostatistics, Gillings School of Global Public Health, University of North 19 Carolina at Chapel Hill, Chapel Hill, NC

21 Corresponding Author:

22 David B. Peden, MD, MS

23 Center for Environmental Medicine, Asthma and Lung Biology

24104 Mason Farm Road, CB 7310, School of Medicine, University of North Carolina at Chapel 25 Hill 
Chapel Hill, NC 27599-7310

\section{ABSTRACT:}

28 Background: We are currently screening human volunteers to determine their sputum

29 polymorphonuclear neutrophil (PMN) response 6 and 24 hours following initiation of exposure to 30 wood smoke particles (WSP). Inflammatory responders ( $\geq 10 \%$ increase in $\% \mathrm{PMN}$ ) are identified

31 for their subsequent participation in mitigation studies against WSP-induced airways

32 inflammation. In this report we compared responder status $(\mathrm{N}=52)$ at both 6 and $24 \mathrm{hr}$ time

33 points to refine/expand its classification, assessed the impact of the GSTM1 genotype, asthma

34 status and sex on responder status, and explored whether sputum soluble phase markers of

35 inflammation correlate with PMN responsiveness to WSP.

36 Results: In the entire cohort, we found a significant, but very small, decrease in FVC and

37 systolic blood pressure immediately following WSP exposure and sputum \%PMNs were

38 significantly increased at 24 hours post exposure, the latter finding was also significantly

39 correlated with sputum IL-1b, IL-6, IL-8, and PMN/mg; a similar response was not found at the 6

40 hour \%PMN response. Blood endpoints in the entire cohort showed a significant increase in

$41 \% \mathrm{PMN}$ and PMN/mg at 6 but not 24 hours. Six-hour responders tended to be 24-hour

42 responders and vice versa, but 24-hour responders also had significantly increased IL-1b, IL-6,

43 IL-8 at 24 hours post WSP exposure. The GSTM1 null genotype significantly $(p<0.05)$ enhanced

44 the \%PMN response at 6 hours in the entire cohort, by $24 \%$ in the 24 -hour responders and not

45 at all in the 6 hours responders. Asthma status enhanced the 24 hour \%PMN response in the

46 entire cohort and in the 6- and 24-hour responders. Sex had no effect on \%PMN response.

47 Conclusions: The 24 hour time point was more informative than the 6 hour time point in

48 optimally defining airway inflammatory responsiveness to WSP exposure. GSTM1 and asthma

49 status are significant effect modifiers of this response. These study design and subject

50 parameters should be considered before enrolling volunteers for proof-of-concept WSP

51 mitigation studies. 


\section{KEYWORDS:}

Wood smoke particle exposure; airway neutrophil response; responder status

\section{BACKGROUND:}

Wood smoke particles (WSP) produced by combustion of biomass contribute to both household and ambient air $\mathrm{PM}_{2.5}$ air pollution, and are associated with exacerbations of asthma, pneumonia, chronic obstructive pulmonary disease and cardiovascular morbidity ${ }^{1}$. Household WSP reach levels $\geq 1000 \mathrm{ug} / \mathrm{m}^{3}$. WSP from wildfires accounts for up to $29 \%$ of ambient fine size (2.5-micron diameter or less) particulate matter (PM) $)_{2.5}$ levels in the US airshed and often abruptly produce ambient air $\mathrm{PM}_{2.5}$ levels $>250 \mathrm{ug} / \mathrm{m}^{3}$, with firefighters often exposed to levels $>750 \mathrm{ug} / \mathrm{m}^{3}{ }^{2}$

We are currently undertaking proof of concept clinical trials of interventions to mitigate the effect of WSP on airway inflammation using a 2-hour controlled exposure to $500 \mathrm{ug} / \mathrm{m}^{3} \mathrm{WSP}$ with exercise sufficient to evoke a minute ventilation of $20 \mathrm{~L} / \mathrm{min} / \mathrm{m}^{2}$ body surface area. We are optimizing these studies by only recruiting volunteers with $a \geq 10 \%$ increase in the polymorphonuclear neutrophil (PMN) content of the sputum differential count following an openlabel screening challenge to WSP. This procedure eliminates non-informative volunteers and is similar to that described by Holz and colleagues ${ }^{34}$ who validated an ozone challenge protocol employed to screen proposed anti-inflammatory agents.

In Holz et al's ozone challenge protocol, only volunteers shown to be responsive to ozone as defined by a $\geq 10 \%$ increase in PMN content of the sputum differential count following a screening challenge to ozone were recruited into the intervention study. Using this doubleblinded, placebo-controlled optimized protocol, they found that both inhaled fluticasone and oral prednisone reduced ozone-induced neutrophilic inflammation. Our group led a second study using Holz et al's validation protocol and found similar results ${ }^{5}$. Subsequently, this protocol has 
82 been employed to screen several agents for their action on acute ozone-induced inflammation.

83 Additionally, we have used this definition of responsiveness to assess the effect of the GSTM1

84 genotype to low level ozone, ${ }^{6}$ and to assess the differences in gene expression between

85 people who are responsive (responders) or non-responsive (non-responders) to ozone ${ }^{7}$.

86 This report documents response of 52 volunteers who have undergone our screening

87 WSP protocol. It expands upon our recent preliminary report of 27 volunteers who had

88 completed this screening protocol, where we observed that $67 \%$ of healthy volunteers were

89 responders, and that the GSTM1 null genotype appeared to increase inflammatory response to

90 WSP as observed 24 hours after challenge ${ }^{8}$. This report expands on these results with a larger

91 sample size which now includes persons with asthma and defines responder status at 2 post

92 exposure time points, namely at 6 hours and 24 hours post challenge. In this report we compare

93 responders and non-responders at both time points, assess the correlation between the 6 and

9424 hour neutrophil response and determine the consistency of responsiveness between

$95 \%$ PMNs and soluble phase markers of airway inflammation. In addition, we report on the effect

96 of WSP exposure on lung function (forced vital capacity (FVC), forced expiratory volume at 1

97 second (FEV1), respiratory rate) and cardiovascular outcomes (heart rate, systolic and diastolic

98 blood pressure), and evaluated these outcomes in persons defined as PMN responders at both

996 and 24 hours post WSP challenge. Finally, we assessed whether the GSTM1 null genotype,

100 asthma status and sex are associated with early and late time point responder status to WSP.

101

102 RESULTS:

103 Following baseline (pre-exposure) assessments of lung function, cardiovascular outcomes and

104 collection of induced sputum, volunteers underwent exposure to $500 \mu \mathrm{g} / \mathrm{m}^{3}$ wood smoke

105 particles for 2 hours, with alternating 15-minute periods of exercise (cycle ergometer) and rest to

106 achieve $25 \mathrm{l} / \mathrm{m}^{2}$ body surface/min minute ventilation. Induced sputum induction was performed 6

107 and 24 hours after initiation of the WSP challenge. 
109 point increase in the percentage of sputum PMNs (\%PMN) at 6 and 24 hours post WSP

110 challenge compared to that at pre-exposure baseline, and the effect of the GSTM1 null

111 genotype on responder status. Additional endpoints assessed the impact of asthma status and

112 sex on 6 and 24 hour-responder statuses, the correlation between sputum soluble phase

113 markers of inflammation (IL-1beta, IL-6, IL-8, TNFalpha) and 6 and 24 hour \%PMN responses

114 and the effect of WSP exposure on lung function (spirometry) and cardiovascular (systolic and 115 diastolic blood pressure, heart rate) outcomes.

\section{Historic baseline vs. Air Challenge control measures in sputum.}

118 As the goal of this screening protocol is to identify persons who are responsive to the 119 inflammatory effect of WSP for subsequent entry into an intervention study, we chose to 120 compare post WSP exposure responses to a pre-exposure baseline, rather than undertake an 121 additional clean air control challenge. In support of this approach, we pooled our own historical 122 data from several air control chamber challenges previously undertaken that involved 2-3 hours 123 of exercise like that employed for this WSP challenge. At pre air challenge baseline, the \%PMN 124 in sputum was $31.7 \pm 3.0 \%$ (mean, SEM, $n=66)$ vs. $29.4 \pm 2.9 \%(n=68)$ at 6 hours post air 125 challenge and $37.0 \pm 8.0 \%(n=10)$ at 24 hours post air challenge $(p=0.7)$. The pre-WSP baseline 126 value was $33.2 \pm 3.2 \% P M N s(n=50)$ and was not statistically different $(p=0.72)$ from the 127 baseline, $6 \mathrm{~h}$ or $24 \mathrm{~h}$ historic time point values. It has also been reported that FVC and FEV1 128 following an air control session are unchanged or increase slightly. These observations support 129 using pre-exposure baseline measures without an air exposure control visit to lower subject 130 burden and increase efficiency for screening responsive volunteers for enrollment into 131 intervention studies. 


\section{Endpoints for the Entire Cohort:}

135 As of April of 2020 (the date at which the protocol was paused due to the COVID-19 pandemic),

13652 volunteers had completed this WSP screening protocol. Table 1 below outlines the

137 demographics of these volunteers.

138 Table 1

\begin{tabular}{|l|l|}
\hline Total Subjects & $\mathrm{N}=52$ \\
\hline Healthy & $\mathrm{N}=40$ \\
\hline Asthmatic & $\mathrm{N}=12$ \\
\hline Female & $\mathrm{N}=26$ (50\%) \\
\hline Race & \\
\hline Asian & $\mathrm{N}=2$ \\
\hline Black & $\mathrm{N}=9$ \\
\hline White & $\mathrm{N}=40$ \\
\hline Other & $\mathrm{N}=1$ \\
\hline BMI & $25.6+0.7$ (range 17.4-38) \\
\hline Age (years) & $27.4+0.8$ (range 19.4-40.3) \\
\hline GSTM1 null & $\mathrm{N}=20$ \\
\hline GSTM1 sufficient & $\mathrm{N}=26$ \\
\hline Refused GSTM1 testing & $\mathrm{N}=6$ \\
\hline
\end{tabular}

140 Figure 1 depicts spirometric and blood pressure endpoints at baseline and immediately after

141 WSP challenge of these volunteers. There is a significant, but very small, decrease in FVC (1A),

142 and systolic blood pressure (1B) immediately following WSP challenge. Of 52 subjects, 50

143 provided at least one sputum sample, with 47 (35 healthy volunteers and 12 with asthma)

144 providing matched baseline and 6-hour timepoint sputum samples. 
Forty-one (30 healthy volunteers and 11 with asthma) subjects were able to provide

146 matched baseline and 24-hour timepoint samples. Figure 2 depicts the sputum \% PMN and

147 soluble phase inflammatory mediators 6 and 24 hours post WSP challenge. Only the \%PMNs

148 in sputum (Figure 2A) are significantly increased by WSP 24 hours after exposure. PMN/mg

149 sputum and sputum levels of IL-1beta, IL-6, IL-8 and TNFalpha (Figure 2, panels B-F

150 respectively) were unchanged by WSP 6 or 24 hours post exposure.

151 We also assessed the correlation between the change from baseline of \%PMNs at 6 and

15224 hours with corresponding change from baseline (expressed as \%change) of the PMN/mg

153 sputum and sputum levels of IL-1beta, IL-6, IL-8 and TNFalpha using Spearman's Rank

154 Correlation as presented in Table 2 below. Only one significant correlation was found at 6 hours

155 (PMN/mg), but 3 cytokine variables and absolute neutrophils were found to be significantly

156 associated at 24 hours post WSP exposure (IL-1beta, IL-6, IL-8, PMN/mg).

157 Table 2:

158

159

160

161

162 We also examined the relationship between change from baseline in sputum \%PMN and

$163 \mathrm{PMN} / \mathrm{mg}$ sputum at 6 and 24 hours with \% change from baseline in FVC, FEV1, systolic and

164 diastolic blood pressure. Of these relationships, the only significant finding $(p<0.05)$ was

24 hour correlations $\%$ change in \% PMNs vs $\%$ change in inflammatory markers

\begin{tabular}{|l|l|l|l|l|l|l|}
\hline & rho & $\mathbf{P}$ & $\mathbf{N}$ & rho & $\mathbf{p}$ & $\mathbf{N}$ \\
\hline IL-1beta & 0.06 & 0.70 & 42 & 0.39 & $\mathbf{0 . 0 2}$ & 36 \\
\hline IL-6 & -0.25 & 0.10 & 43 & 0.36 & $\mathbf{0 . 0 4}$ & 34 \\
\hline IL-8 & -0.08 & 0.60 & 43 & 0.37 & $\mathbf{0 . 0 2}$ & 37 \\
\hline TNFalpha & 0.03 & 0.80 & 43 & 0.09 & 0.60 & 36 \\
\hline PMN/mg & 0.35 & $\mathbf{0 . 0 2}$ & 47 & 0.60 & $\mathbf{0 . 0 1}$ & 41 \\
\hline
\end{tabular}

6 hour correlations

.

.


165 between the \%change in PMN/mg sputum at 24 hours and \% change in FVC $(r=-0.36)$; we did 166 observe a trend $(p<0.1)$ between PMN/mg sputum at 24 hours and \% change in FEV1 $(r=-031)$.

168 Table 3

169

\begin{tabular}{|l|l|l|l|l|l|l|l|l|l|}
\hline \multirow{2}{*}{ Endpoint } & \multicolumn{3}{l}{ Baseline } & \multicolumn{3}{l}{ 6hr post } & \multicolumn{3}{l|}{ 24 hr post } \\
\cline { 2 - 12 } & Mean & SE & N & Mean & SE & N & Mean & SE & N \\
\hline \%PMN blood & 54.90 & 1.08 & 53 & $61.65^{\star}$ & 0.88 & 52 & 57.21 & 1.09 & 53 \\
\hline PMN 106/ul blood & 3.10 & 0.12 & 53 & $4.52^{*}$ & 0.14 & 52 & $3.50^{*}$ & 0.16 & 53 \\
\hline IL-8 blood & 13.60 & 1.60 & 48 & 9.59 & 0.75 & 50 & 10.88 & 0.88 & 43 \\
\hline IL-6 blood & 0.80 & 0.10 & 47 & 1.01 & 0.11 & 50 & 0.82 & 0.11 & 43 \\
\hline IL-1 beta blood & 0.10 & 0.02 & 41 & 0.08 & 0.02 & 49 & 0.06 & 0.01 & 38 \\
\hline TNFalpha blood & 2.36 & 0.21 & 48 & 2.09 & 0.17 & 50 & 2.15 & 0.15 & 43 \\
\hline CRP blood & 2.60 & 0.40 & 45 & 2.60 & 0.42 & 44 & 2.67 & 0.46 & 45 \\
\hline
\end{tabular}

We also measured blood endpoints from volunteers at 6 and 24 hours post WSP

172 differential count (expressed as percent of total nucleated cells), and levels of IL-1beta, IL-6, IL-

173 8, TNFalpha and C-reactive protein (CRP). The \% PMN and PMN/mg levels were significantly

$174(p<0.05)$ increased above baseline at 6 hours, but not 24 hours. All other measures were

175 unchanged across all the cohorts.

177 Endpoints of Responders defined by 6-hour \%PMN sputum response:

178 Forty-seven volunteers provided sputum samples at baseline and six hours after initiation of

179 WSP challenge. Of these volunteers, 30 (64\%) were responsive to WSP, as defined by a $\geq 10 \%$

180 point increase in sputum \%PMNs. Figure 3 depicts spirometric and blood pressure endpoints at

181 baseline and immediately after WSP challenge in responders and non-responders. There is a

182 significant, but very small, decrease in FVC (3A), and systolic blood pressure (3B) immediately 
183 following WSP challenge in responders but not in the non-responders. Figure 4 depicts the

184 airway inflammatory response (cells and soluble phase mediators) to woodsmoke particles of 6

185 hour responders $(\mathrm{N}=30)$ and non-responders $(\mathrm{N}=17)$. On average, 6 -hour responders also had

186 significantly $(\mathrm{p}<0.05)$ increased \%PMN responses at 24 hours, but no other inflammatory

187 endpoints demonstrated this effect. Systemic inflammatory endpoints were similar when

188 stratified based on airway response at 6 hours (data not shown).

190 Endpoints of Responders defined by 24-hour \%PMN sputum response:

191 Forty-one volunteers provided sputum samples at baseline and 24 hours after initiation of WSP

192 challenge. Of these volunteers, $28(68 \%)$ were responsive to WSP, as defined by a $\geq 10 \%$

193 increase in sputum \%PMNs. Figure 5 depicts spirometric and blood pressure endpoints at

194 baseline and immediately after WSP challenge of volunteers. There is a significant, but very

195 small, decrease in FVC (3A), and systolic blood pressure (3B) immediately following WSP

196 challenge in responders but not in non-responders Figure 6 depicts the airway inflammatory

197 response (cells and soluble phase mediators) to woodsmoke particles of 24-hour responders

$198(\mathrm{~N}=28)$ and non-responders $(\mathrm{N}=13)$. On average, 24-hour responders had significantly

199 increased \%PMN and PMN/mg responses at both 24 and 6 hours. Interestingly, and unlike 6-

200 hour responders, 24-hour responders showed significantly increased levels of several pro-

201 inflammatory mediators at 24 hours post WSP exposure (IL-1b, IL-6, IL-8). Systemic

202 endpoints were again similar when stratified based on airway response at 24 hours (data not 203 shown).

205 Effect of GSTM1 genotype, asthma status and sex on the airway inflammatory response

206 to WSP: We assessed the role of GSTM1 genotype, asthma status and sex on the sputum

$207 \%$ PMN, PMN/mg sputum and sputum IL-1beta, IL-6, IL-8 and TNFalpha response to WSP at 6

208 and 24 hours using the entire cohort, the 6-hour responder cohort and the 24-hour responder 
209 cohort. We used linear regression modeling approaches in which a given response was

210 expressed as $\%$ of baseline, where, $R_{\text {outcome }}=\frac{\text { postbaselineoutcome }}{\text { baselineoutcome }}$, as our main response

211 variable, with a responder defined as $R \geq 1.1$ (equivalent to a $10 \%$ increase from baseline in

$212 \% \mathrm{PMN})$. For instance, $R_{\% P M N 24}=\frac{\% P M N a t 24 \text { hourspost }}{\% \text { PMNatbaseline }}$. To determine if there is a difference in post

213 baseline versus baseline, we first fit an intercept only model, where the response is defined as

$214 R_{\text {outcome }}-1$. Thus, the hypothesis test for WSP effect is equivalent to testing whether the ratio

215 is statistically different from 1 , or equivalently, a t-test to test if $\hat{\beta}_{0}=0$ in an intercept only model.

216 We next expanded our regression model to examine if there are any differences in the ratio

217 between the status of GSTM1, Asthmatics, and responder status (with beta1 in Table 4

218 indicates the slope for those variables). We also fit the regression model to allow for other

219 covariates of interest that might influence the response variable, as described fully in Methods.

220 Using this technique, we showed the effect of GSTM1, Asthma and Sex on sputum

$221 \%$ PMNs as seen in Table 4 below. We found that the GSTM1 null genotype significantly

$222(p<0.05)$ enhanced only the \%PMN variable and none of the other inflammatory variables as

223 follows: in the entire cohort (Table 4a) at 6 hours post WSP exposure; by $24 \%$ in the 24 -hour

224 responders (Table 4b); and not at all in the 6-hour responders (Table 4c). Likewise, asthma

225 status enhanced just the \%PMN variable and did so at 24 hours in each of the cohorts (entire

226 cohort; 24-hour responders; 6-hour responders), with sex having no effect on this measure.

227 These factors had no effect on any of the other inflammatory outcomes (data not shown). 
234 Table 4a. GSTM1, Asthma status, and Sex effect on cytokine and neutrophil response at 24

235 hour and 6 hours in the Entire Cohort

\begin{tabular}{|c|c|c|c|c|c|c|c|c|c|}
\hline & \multicolumn{3}{|c|}{ GSTM1 } & \multicolumn{3}{|c|}{ Asthma status } & \multicolumn{3}{|c|}{ Sex } \\
\hline & $\mathbf{N}$ & beta1/SE & $\begin{array}{c}p \\
<0.0 \\
5\end{array}$ & $\mathbf{n}$ & beta1/SE & $\begin{aligned} & p \\
&<0.0 \\
& 5\end{aligned}$ & $\mathbf{n}$ & beta1/SE & $\begin{array}{c}p \\
<0.0 \\
5\end{array}$ \\
\hline $\begin{array}{l}\text { \%PMN } \\
@ 24 h r\end{array}$ & 38 & $\begin{array}{c}- \\
0.602 / 0.500\end{array}$ & - & 44 & $1.170 / 0.501$ & * & 44 & $\stackrel{-}{-}$ & - \\
\hline \%PMN @6hr & 42 & $\begin{array}{c}- \\
0.514 / 0.311\end{array}$ & * & 49 & $0.755 / 0.646$ & - & 49 & $\stackrel{-}{-}$ & - \\
\hline
\end{tabular}

236

237

238

239

240

241

242

243

244

245

246

247

248

249

Table 4b. GSTM1, Asthma status, and Sex effect on cytokine and neutrophil response at 24 hour and 6 hours in the 24 hour - Responders

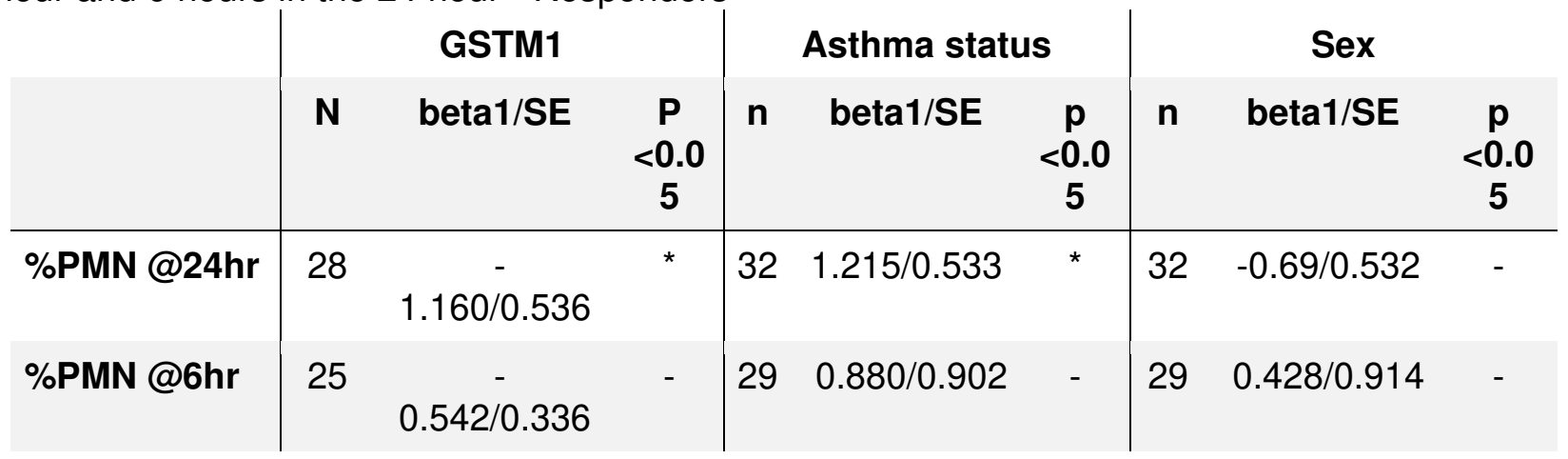

Table 4c. GSTM1, Asthma status, and Sex effect on cytokine and neutrophil response at 24 hour and 6 hours in the 6 hour - Responders

\begin{tabular}{|c|c|c|c|c|c|c|c|c|c|}
\hline & $\mathbf{N}$ & beta1/SE & $\begin{array}{c}P \\
<0.0 \\
5\end{array}$ & $\mathbf{N}$ & beta1/SE & $\begin{array}{c}p \\
<0.0 \\
5\end{array}$ & $\mathbf{N}$ & beta1/SE & $\begin{array}{c}P \\
<0.0 \\
5\end{array}$ \\
\hline \%PMN @24hr & 23 & $\begin{array}{c}- \\
0.715 / 0.703\end{array}$ & - & 28 & $1.587 / 0.636$ & * & 28 & $\begin{array}{c}- \\
0.900 / 0.685\end{array}$ & - \\
\hline \%PMN @6hr & 27 & $\begin{array}{c}- \\
0.385 / 0.329\end{array}$ & - & 33 & $0.820 / 0.816$ & - & 33 & $0.090 / 0.804$ & - \\
\hline
\end{tabular}

\section{DISCUSSION:}

This protocol was developed to screen and identify volunteers who are inflammatory responders to WSP for their entry into subsequent early phase mitigation studies against WSP- 
250 induced airways inflammation. We used our previously published inflammatory responder 251 classification that identified responders as having a greater than $10 \%$-point increase from pre-

252 exposure baseline in sputum \%PMN at 6 hours, but here, extended our responder classification

253 to examine the utility of the 24 hour \%PMN time point. We further the effect of the GSTM1

254 genotype, asthma status and sex on responder status, and explored whether WSP exposure

255 induced changes in spirometry and blood pressure outcomes and if sputum inflammatory

256 mediators were associated with \%PMN responder status.

257 Within the overall cohort, we found that sputum \%PMN were significantly elevated at 24

258 hours after WSP exposure. Circulating PMN cell count was also increased at 6 and 24 hours,

259 but no other sputum markers of inflammation, namely soluble phase mediators and PMN/mg,

260 were increased after WSP exposure. When we stratified persons on the basis of neutrophilic

261 airway responsiveness to WSP, we found that $64 \%$ were responders at 6 hours and $68 \%$ were

262 responders at 24 hours. We also found that the FVC and systolic blood pressure were

263 decreased in the overall cohort and in 6 and 24-hour responder cohorts.

264 As the goal of the screening protocol is to optimally identify inflammatory responders for

265 intervention studies, we compared results of volunteers defined as responsive using the 6-hour

266 sputum to those defined on the basis of the 24-hour sputum. While the \% PMNs were observed

267 to be significantly increased in the 6 hour and 24-hour responder cohorts, we also observed that

268 all of the sputum cytokines except for TNFalpha were increased at 24 hours and not at 6 hours.

269 When we examined the correlation between all the sputum inflammatory markers (cytokines

270 and PMN $/ \mathrm{mg}$ ) with change in \%PMNs at 6 and 24 hours, we found that while the \%PMNs and

$271 \mathrm{PMN} / \mathrm{mg}$ sputum significantly correlated in both responder groups, the inflammatory cytokines

272 were significantly correlated only in the 24-hour responder group. We also assessed the effect

273 of the GSTM1 genotype, asthma status and sex on the airway inflammatory response to WSP

274 and found that GSTM1 and Asthma status both significantly impacted \%PMN responses in the

275 overall cohort as well as those classified as responders at both 6 and 24 hours. 
We further observed that the individual airway inflammatory markers had the highest

277 degree of correlation with each other 24 hours rather than 6 hours after initiation of WSP

278 exposure. In our estimation, 24 hours rather than 6 hours, is the more informative timepoint for

279 establishing inflammatory responder status to WSP exposure in order to enter phase I/II proof of

280 concept mitigation studies. We have conducted previous intervention studies with other air

281 pollutants like ozone ${ }^{5}$ where examining the impact of the GSTM1 genotype on the airway

282 inflammatory response has revealed informative information in terms of responder status at 6 vs

28324 hours post exposure. ${ }^{6}$ Like the results reported here with WSP, we have previously found

284 that that the GSTM1 genotype is an important determinant of response to ozone, that

285 significantly impacts the inflammatory responder status of that inhaled pollutant. Indeed, in a

286 previous study of 59 volunteers assessed for the effect of $0.06 \mathrm{ppm}$ ozone on lung function and

287 inflammation, we did not see a significant effect of GSTM1 when \%PMNs were expressed as a

288 continuous outcome variable in GSTM1 null vs sufficient volunteers ${ }^{6}$. However, when we

289 reanalyzed those data in a follow study, we observed that the likelihood of being an

290 inflammatory responder to ozone, defined using similar criteria to what we employed in this

291 study, was 13-fold higher in the GSTM1 null population than in the sufficient population ${ }^{7}$. Our

292 group have also performed studies that suggest that inflammatory responders to air pollutants

293 like ozone possess unique genomic signatures and microRNA expression profiles that mediate

294 important biological processes like immune cell trafficking, and immune and inflammatory cell

295 function. ${ }^{89}$ These studies, together with the data presented here on WSP, suggest that

296 identification of risk factors or testing of interventions to mitigate airway responses to air

297 pollutants like wood smoke particles, should focus on inflammatory responsive individuals,

298 where effect modifiers like GSTM1 genotype and asthma status need to be considered when

299 evaluating respiratory outcomes.

300

301 CONCLUSIONS: 
302 Our data suggest that 24 hours rather than 6 hours post exposure, is the more informative time 303 point for defining airway inflammatory responders to wood smoke particle exposure. Further,

304 GSTM1 and asthma status should be considered important effect modifiers of responder status.

\section{METHODS:}

307 A total of 52 subjects (26 male, 40 healthy, 12 asthmatics), aged 18-45 years completed the 308 WSP screening protocol (Table 1, subject demographics). Fifty subjects provided at least one 309 sputum sample; 47 (35 healthy; 12 asthmatics) provided matched baseline and 6-hour timepoint 310 sputum samples; and 41 (30 healthy; 11 asthmatics) provided matched baseline and 24-hour 311 timepoint sputum samples. All subjects were non-smokers with no acute respiratory illness in 312 the prior 4 weeks, and no current allergic rhinitis symptoms. Asthmatics had mild to moderate 313 physician diagnosed asthma, a positive methacholine challenge test and were not on oral 314 corticosteroid therapy. The GSTM1 genotype was determined by buccal swab analysis using 315 methods previously described ${ }^{6}$. Twenty subjects were GSTM1null, twenty-six were GSTM1 316 sufficient, with six refusing GSTM1 genotyping. Written consent was obtained from all

317 participants, and the study was approved by the University of North Carolina Institutional 318 Review Board.

320 Details of the WSP exposure protocol are described previously. ${ }^{10}$ In brief, baseline induced 321 sputum samples were obtained prior to the WSP chamber visit and at 6 and 24 hours following 322 WSP exposure. The WSP chamber used wood smoke generated by heating red oak wood on 323 an electric heating element. Subjects were exposed to $500 \mu \mathrm{g} / \mathrm{m}^{3}$ WSP over a 2-hour period 324 with alternating 15-minute periods of rest and exercise on a cycle ergometer at a level sufficient 325 to produce a minute ventilation of $20 \mathrm{~L} / \mathrm{min} / \mathrm{m}^{2}$ body surface area. Induced sputum was collected 326 and processed according to previously published methods. ${ }^{10}$ The primary endpoints were 327 sputum percent neutrophils (\%PMNs) at 6 and 24 hours post- initiation of the WSP exposure 
328 compared to baseline samples. Consistent with previous studies at our center, 1011 inflammatory

329 responders were defined as those who experienced $a \geq 10$ percentage point increase in sputum

$330 \%$ PMN. Measures of lung function (spirometry), cardiovascular status (blood pressure, heart

331 rate) sputum soluble markers and serum inflammatory markers were also recorded at baseline

332 and 6 and 24 hour post WSP exposure time points.

\section{Statistical Analyses:}

We compared the effect of woodsmoke particle (WSP) exposure 6 and 24 hours after

336 beginning the 2-hour exposure challenge to baseline lung function, sputum and blood

337 measures. We analyzed these data by fitting a mixed model as implemented in GraphPad Prism

338 8.0. This mixed model uses a compound symmetry covariance matrix and is fit using Restricted

339 Maximum Likelihood (REML). In the absence of missing values, this method gives the same $\mathrm{P}$

340 values and multiple comparisons tests as repeated measures ANOVA. In the presence of

341 missing values (missing completely at random), the results can be interpreted like repeated

342 measures ANOVA.

343 To assess the effect of GSTM1, Asthma and sex on sputum inflammatory measures, we

344 assessed the sputum outcomes of interest measured at baseline, 6 hours after exposure

345 commenced, and 24 hours after exposure commenced. To investigate if wood smoke (WSP)

346 had an effect on subjects, for endpoint X, we used the ratio, defined as $R_{x}=\frac{X_{\_} \text {post }}{X_{\_} p r e}$. For instance,

$347 R_{\% P M N 24}=\frac{\% P M N a t 24 \text { hours }}{\% \text { PMNatbaseline }}$. For the form of the outcome variable, we also considered the

348 differences from the baseline, as well as various transformations, e.g., log transformation. For

349 modeling, we selected the ratio form of our outcomes based on the QQ plots to assess the

350 model assumptions. ${ }^{12}$ The hypothesis test for WSP effect is to test whether the ratio is

351 statistically different from 1 . We fit the following regression model,

$$
Y=\beta_{0}
$$


353 where $Y=R_{P M N}-1$ is the response variable, and $\beta_{0}$ is the intercept. The $\mathrm{p}$-value for testing

$354 \beta_{0}=0$ is equivalent of testing $R_{P M N}=1$, i.e., if there is a significant change in outcome at post 355 exposure from pre-exposure.

356 We then employed the regression model to examine whether GSTM1 status or Asthma 357 status have a significant effect on the ratio of interest. We fit the following model,

$$
R_{P M N}=\beta_{0}+\beta_{1} * x_{\text {covar }}
$$

359 where $x_{\text {covar }}$ is the covariate of interest. For GSTM1 status, we will use GSTM1-Null (GSTM1-)

360 as the reference group $\left(x_{\text {covar }}=1\right.$ ifGSTM1+, elsex $\left.x_{\text {covar }}=0\right)$. For Asthma status, we will use

361 Healthy as the reference group $\left(x_{\text {covar }}=1\right.$ if Asthmatic, else $\left.x_{\text {covar }}=0\right)$. The $\mathrm{p}$-value for $\beta_{1}$ tests

362 whether $\hat{\beta_{1}}=0$, i.e., if there is a significant change in the outcome between the covariate

363 levels. We fit the models using $\operatorname{lm}()$ in $\mathrm{R} 3.6 .1^{12}$. 
LIST OF ABBREVIATIONS

WSP wood smoke particles; GSTM1 glutathione S transferase mu 1; PMN polymorphonuclear

367 neutrophil; FVC forced vital capacity; FEV1 forced expiratory volume in 1 second; IL interleukin;

368 TNFalpha tumor necrosis factor alpha; ug $/ \mathrm{m}^{3}$ microgram per cubic meter; BMI body mass index;

369 ANOVA analysis of variance; $1 / \mathrm{m}^{2}$ liters per square meter

\section{DECLARATIONS}

\section{Ethics approval and consent to participate}

373 This study (IRBIS 15-1775 titled "To identify persons who are susceptible to WSP-induced

374 inflammation and examine the role of GSTM1 and other factors in this susceptibility") was

375 reviewed and approved by the Office of Human Research Ethics of the University of North

376 Carolina at Chapel Hill (the UNC-CH IRB). All volunteers provided informed consent prior to

377 participation in this study.

\section{Consent for publication}

380 All data are mean and standard error of the mean with no individually identifiable personal data

381 being reported

\section{Availability of data and materials}

384 The datasets used and/or analyzed during the current study are available from the

385 corresponding author on reasonable request. Additionally, this study is listed in

386 ClinicalTrials.gov (NCT02767973), and data will be posted in that database upon completion of

387 the study. Where informed consent was given, all unused de-identified biospecimens will be

388 stored and curated in the CEMALB Biorepository (IRB\# 05-2528) for potential future use. 
391 Competing interests

392 Authors

393 "The authors declare that they have no competing interests"

395 Funding

396 Research reported in this publication was primarily supported by the National Institute of

397 Environmental Health Sciences of the National Institutes of Health under award number

398 R01ES025124 and Assistance Agreement no. 83578501-0 awarded by the US Environmental

399 Protection Agency (EPA) to the University of North Carolina. The content is solely the

400 responsibility of the authors and does not necessarily represent the official views of the National

401 Institutes of Health or the Environmental Protection Agency. The EPA does not endorse any

402 products or commercial services mentioned in this publication. L.Z. is supported by grant

403 5T32ES007018. M.L.H and A.J.B. are supported by grant R01HL135235. H.Z. is supported by

404 grant P30ES010126. The NIEHS, NHLBI, and EPA had no role in the design of the study, or

405 collection, analysis, and interpretation of data or in writing the manuscript.

406 All sources of funding for the research reported should be declared. The role of the funding

407 body in the design of the study and collection, analysis, and interpretation of data and in writing

408 the manuscript should be declared.

\section{Authors' contributions}

411 DBP conceived the study, oversaw regulatory and medical aspects of the study, and was

412 involved in data collection, data analysis and manuscript preparation. LZ conducted statistical

413 data analysis and contributed to manuscript preparation. AJB developed the IRB application and

414 was involved in medical oversight of the study and data collection. MA was involved in IRB

415 preparation and data collection. MLH was involved in medical oversight of the study and data

416 collection in data collection. KHM was involved in IRB preparation and data collection. TLN was 
417 contributed to medical oversight of the study and data collection. HW oversaw sample

418 processing and contributed to data collection and analysis. $\mathrm{HZ}$ oversaw statistical analysis of

419 the data and contributed to manuscript preparation. NEA was involved in manuscript

420 preparation and all aspects of sample collection, laboratory processing and analysis.

422 Acknowledgements

423 Not applicable

425 Authors' information (optional)

426 Not applicable

\section{REFERENCES}

429 1. Oluwole, O., Arinola, G.O., Huo, D. et al. Household biomass fuel use, asthma

430 symptoms severity, and asthma underdiagnosis in rural schoolchildren in Nigeria: a cross-

431 sectional observational study. BMC Pulm Med 17, 3 (2017). https://doi.org/10.1186/s12890-016-

$432 \quad 0352-8$

433 2. Aguilera, R., Corringham, T., Gershunov, A. et al. Wildfire smoke impacts respiratory

434 health more than fine particles from other sources: observational evidence from Southern

435 California. Nat Commun 12, 1493 (2021). https://doi.org/10.1038/s41467-021-21708-0

436 3. Holz, O., Biller, H., Mueller, M. et al. Efficacy and safety of inhaled calcium lactate

437 PUR118 in the ozone challenge model - a clinical trial. BMC Pharmacol Toxicol 16, 21 (2015).

438 https://doi.org/10.1186/s40360-015-0021-1

439 4. Olaf Holz, Ruth Tal-Singer, Frank Kanniess, Kathy J. Simpson, Anthony Gibson, Rupert

440 S. J. Vessey, Stanislawa Janicki, Helgo Magnussen, Rudolf A. Jörres and Kai Richter Validation

441 of the Human Ozone Challenge Model as a Tool for Assessing Anti-Inflammatory Drugs in Early

442 Development J Clin Pharmacol 2005 45: 498 DOI: 10.1177/0091270004273527

$443 \quad 5 . \quad$ Neil E. Alexis, John C. Lay, Angela Haczku, Henry Gong, William Linn, Milan J.

444 Hazucha, Brad Harris, Ruth Tal-Singer, David B. Peden Fluticasone Propionate Protects 
445 against Ozone-Induced Airway Inflammation and Modified Immune Cell Activation Markers in

446 Healthy Volunteers EHP June 1, 2008, https://doi.org/10.1289/ehp.10981

447 6. Neil E. Alexis, Haibo Zhou, John C Lay, Bradford Harris, Michelle L Hernandez, sui-

448 Shan Lu, Philip A. Bromberg, David Diaz-Sanchez, Robert B. Devlin, Steven R. Kleeberger,

449 David B. Peden The Glutathione-S-Transferase Mu 1 null genotype modulates ozone-induced

450 airway inflammation in humans J Allergy Clin Immunol. 2009 Dec; 124(6): 1222-1228.e5. doi:

$451 \quad$ 10.1016/j.jaci.2009.07.036

452 7. Neil E. Alexis, John C. Lay, Haibo Zhou, Chong S. Kim, Michelle L. Hernandez, Howard 453 Kehrl, Milan J. Hazucha, Robert B. Devlin, David Diaz-Sanchez, David B. Peden. The

454 glutathione-S-transferase mu 1 (GSTM1) null genotype and increased neutrophil response to 455 low-level ozone (0.06 ppm) JACl, 2013 Feb;131(2):610-2

456 8. Rebecca C. Fry, Julia E. Rager, Rebecca Bauer, Elizabeth Sebastian, David B. Peden, 457 Ilona Jaspers, Neil E. Alexis Air toxics and epigenetic effects: ozone altered microRNAs in the 458 sputum of human subjects Am J Physiol Lung Cell Mol Physiol. 2014 Jun 15; 306(12): L1129_

459 L1137. doi: 10.1152/ajplung.00348.2013

460 9. Fry RC, Rager JE, Zhou H, Zou B, Brickey JW, Ting J, Lay JC, Peden DB, Alexis NE. 461 Individuals with increased inflammatory response to ozone demonstrate muted signaling of 462 immune cell trafficking pathways. Respir Res. 2012 Oct 3;13(1):89.

463 10. Allison J. Burbank, Michelle L. Hernandez, Katherine H. Mills, Ethan M. Alt, Haibo Zhou, 464 David B. Peden Characterizing Airway Inflammatory Responses to Wood-Smoke Inhalation 465 JACI 2019 Volume 143DOI: https://doi.org/10.1016/j.jaci.2018.12.073

466 11. Madeline A Dillon, Bradford Harris, Michelle L Hernandez, Baiming Zou, William Reed, 467 Philip A Bromberg, Robert B Devlin, David Diaz-Sanchez, Steven Kleeberger, Haibo Zhou, John 468 C Lay, Neil E Alexis, and David B Peden Enhancement of systemic and sputum granulocyte 469 response to inhaled endotoxin in people with the GSTM1 null genotype. Occup Environ Med. 470 2011 Oct; 68(10): 10.1136/oem.2010.061747. doi: 10.1136/oem.2010.061747 
471 12. R Core Team (2019). R: A language and environment for statistical computing. $R$

472 Foundation for Statistical Computing, Vienna, Austria. URL https://www.R-project.org/To assess

473 the effect of GSTM1 genotype, asthma status and sex on sputum

474

475 FIGURES, TABLES AND ADDITIONAL FILES

476 Table Titles and Legends

477 Table 1 Title: Subject Demographics.

478 Table 2 Title: Correlations between sputum \%PMNs and other sputum inflammatory endpoints

479 Table 3 Title: Systemic Endpoints of Entire Cohort at 6 and 24 hours Post WSP Exposure

480 Table 3 Legend: Descriptive data (mean, standard error and $\mathrm{n}$ for available sample) for each

481 systemic endpoint of the overall cohort at baseline, 6 and 24 hours after two-hour controlled

482 exposure to $500 \mathrm{ug} / \mathrm{m}^{3} \mathrm{WSP}$. Cytokines in blood are measured as $\mathrm{pg} / \mathrm{ml}$. Asterisk denotes

$483 p<0.05$ vs. baseline values, which were present only for the circulating PMN differential count

484 (\%PMN) and absolute PMN levels (10\%/ul blood)

485 Table 4 Title: Regression analyses for GSTM1, asthma and sex on \%PMN:

486 Figure Titles and Legends

487 Figure 1 Title: Spirometric and Blood Pressure response to WSP in the overall cohort

488 Figure 1 Legend: Spirometric and blood pressure endpoints at baseline and immediately after

489 WSP challenge. Fifty subjects provided at least one sputum sample, with 47 (35 healthy

490 volunteers and 12 with asthma) providing matched baseline and 6-hour timepoint sputum

491 samples

492 Figure 2 Title: Inflammatory Response to Woodsmoke Particles of the Entire Cohort

493 Figure 2 Legend: Sputum \% PMN and soluble phase inflammatory mediators 6 and 24 hours

494 post WSP challenge.

495 Figure 3 Title: Spirometric and blood pressure endpoints at baseline and immediately after WSP 496 challenge of responsive volunteers as defined by \%PMNs at 6 hours. 
497 Figure 3 Legend: Spirometric and blood pressure endpoints at baseline and immediately after

498 WSP challenge in responders and non-responders

499 Figure 4 Title: Inflammatory Response to Woodsmoke Particles of responsive volunteers as

500 defined by \%PMNs at 6 hours.

501 Figure 4 Legend: The airway inflammatory response (cells and soluble phase mediators) to

502 woodsmoke particles of 6 -hour responders $(\mathrm{N}=30)$ and non-responders $(\mathrm{N}=17)$

503 Figure 5 Title: Spirometric and blood pressure endpoints at baseline and immediately after WSP

504 challenge of responsive volunteers as defined by \%PMNs at 24 hours

505 Figure 5 Legend: Spirometric and blood pressure endpoints at baseline and immediately after

506 WSP challenge of volunteers

507 Figure 6 Title: Inflammatory Response to Woodsmoke Particles of responsive volunteers as

508 defined by \%PMNs at 24 hours

509 Figure 6 Legend: The airway inflammatory response (cells and soluble phase mediators) to

510 woodsmoke particles of 24-hour responders $(\mathrm{N}=28)$ and non-responders $(\mathrm{N}=13)$. 
Figures
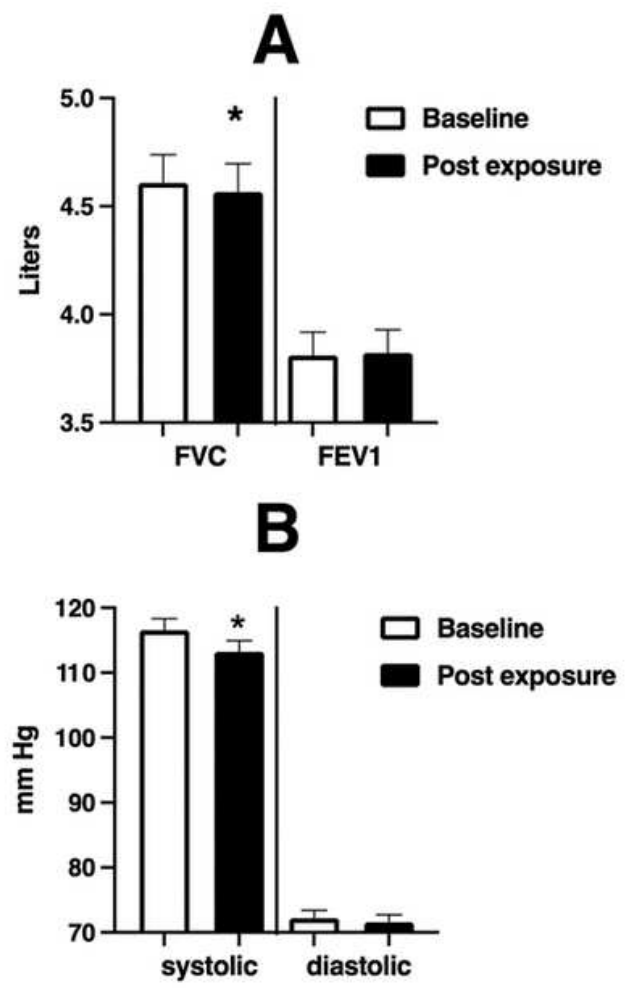

\section{Figure 1}

Spirometric and Blood Pressure response to WSP in the overall cohort Spirometric and blood pressure endpoints at baseline and immediately after WSP challenge. Fifty subjects provided at least one sputum sample, with 47 (35 healthy volunteers and 12 with asthma) providing matched baseline and 6-hour timepoint sputum samples 
A

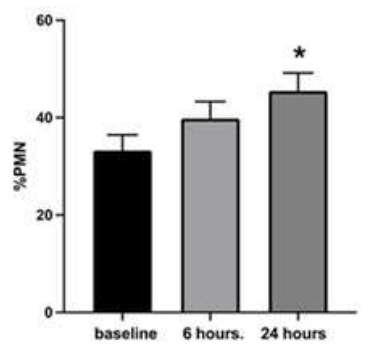

D

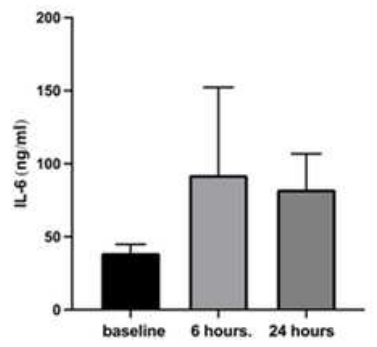

B

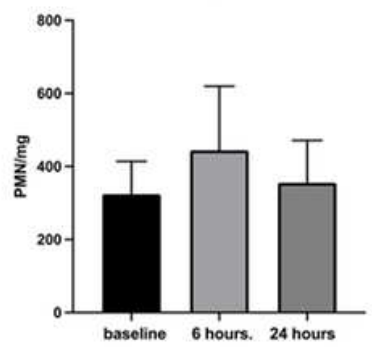

E

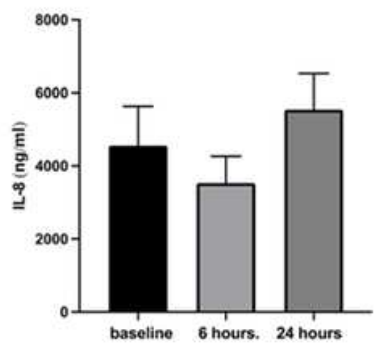

C

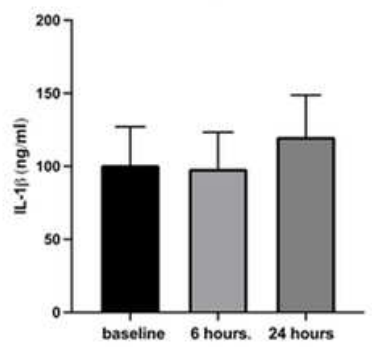

$\mathbf{F}$

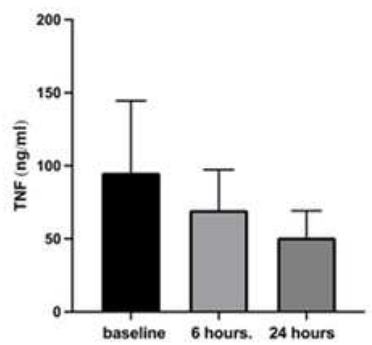

Figure 2

Inflammatory Response to Woodsmoke Particles of the Entire Cohort Sputum \% PMN and soluble phase inflammatory mediators 6 and 24 hours post WSP challenge.
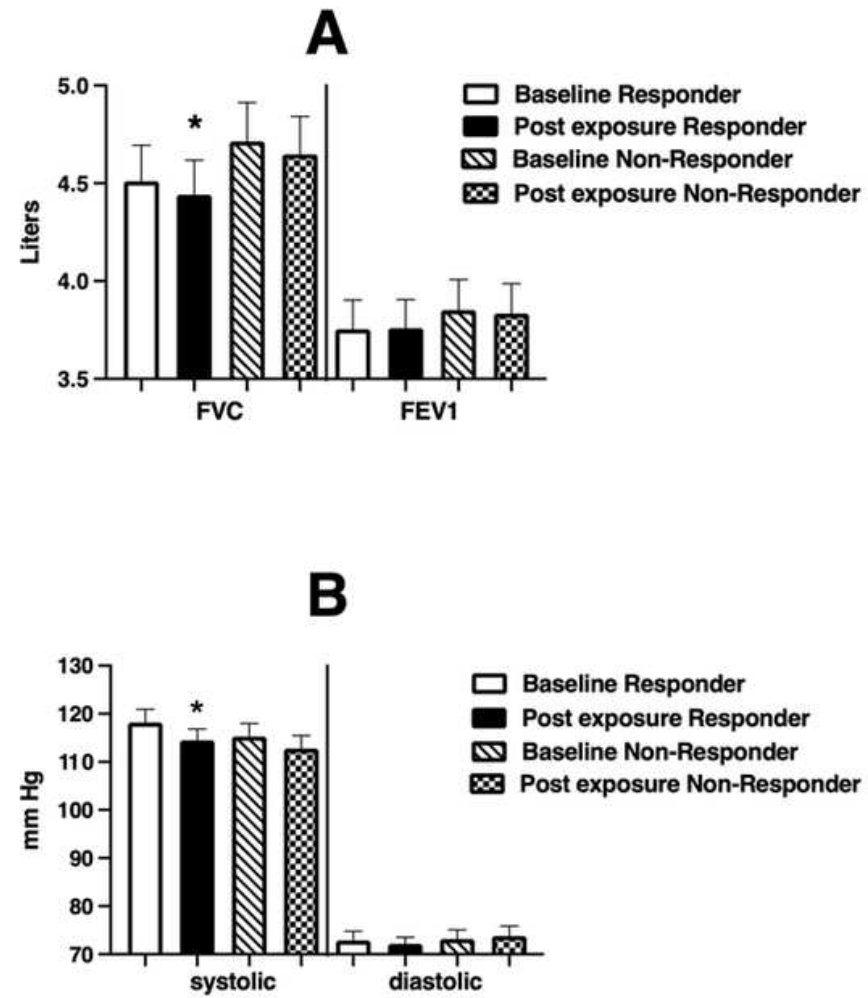
Figure 3

Spirometric and blood pressure endpoints at baseline and immediately after WSP challenge of responsive volunteers as defined by \%PMNs at 6 hours. Spirometric and blood pressure endpoints at baseline and immediately after WSP challenge in responders and non-responders

A

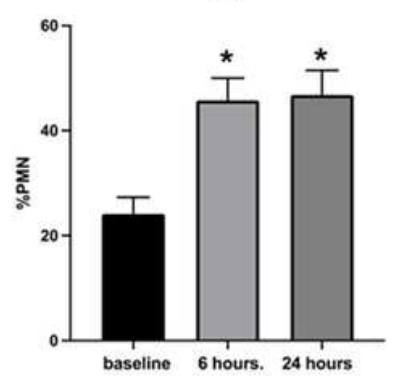

D

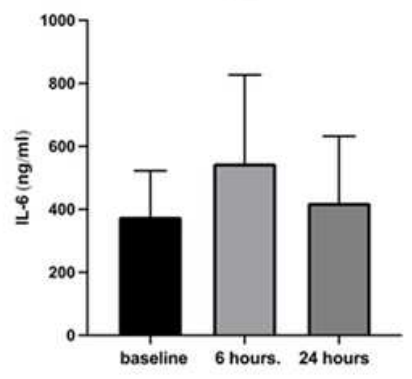

B

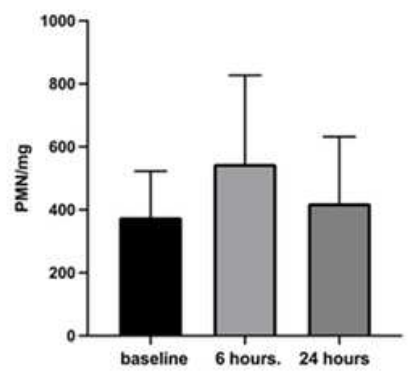

E

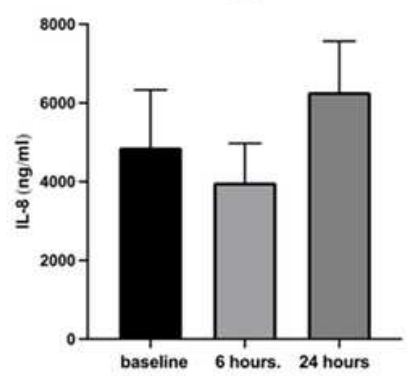

C

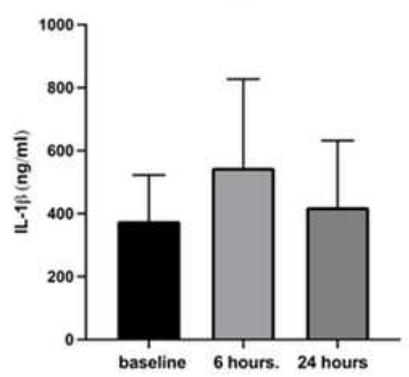

$\mathbf{F}$

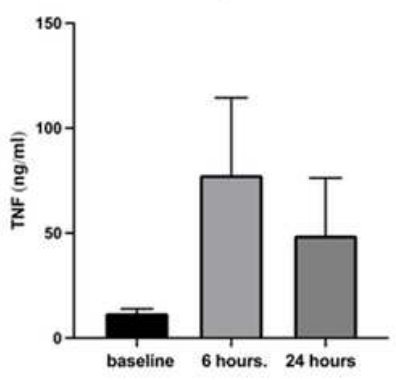

Figure 4

Inflammatory Response to Woodsmoke Particles of responsive volunteers as defined by \%PMNs at 6 hours. The airway inflammatory response (cells and soluble phase mediators) to woodsmoke particles of 6-hour responders $(\mathrm{N}=30)$ and non-responders $(\mathrm{N}=17)$ 

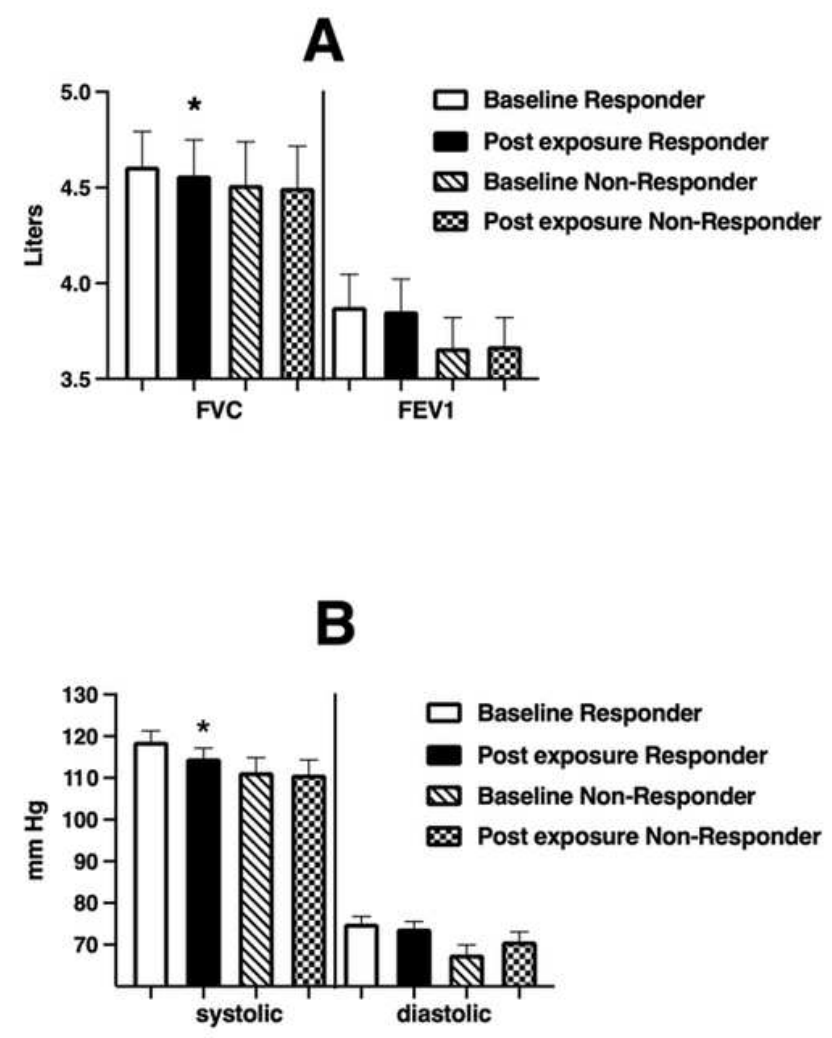

Figure 5

Spirometric and blood pressure endpoints at baseline and immediately after WSP challenge of responsive volunteers as defined by \%PMNs at 24 hours Spirometric and blood pressure endpoints at baseline and immediately after WSP challenge of volunteers 
A

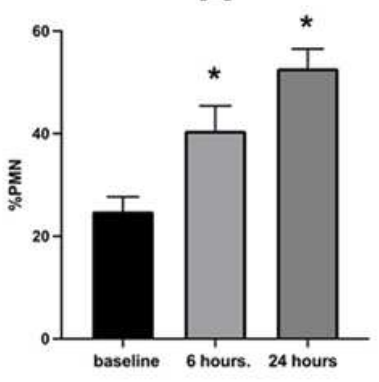

D

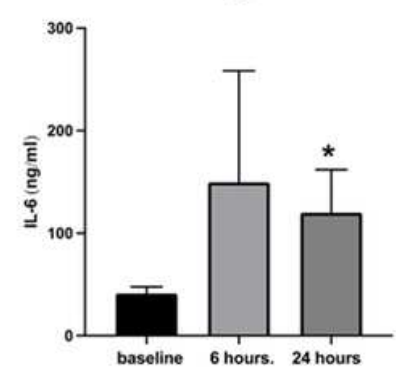

B

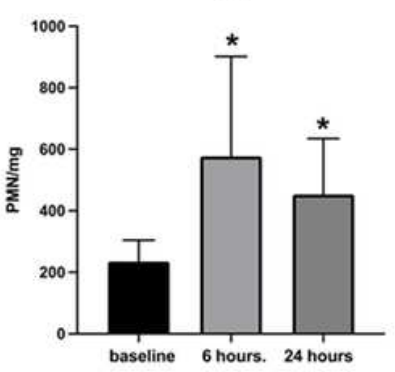

$\mathbf{E}$

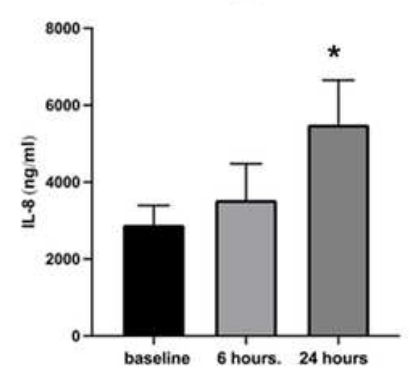

C

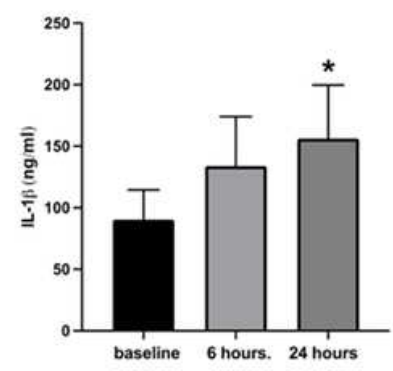

$\mathbf{F}$

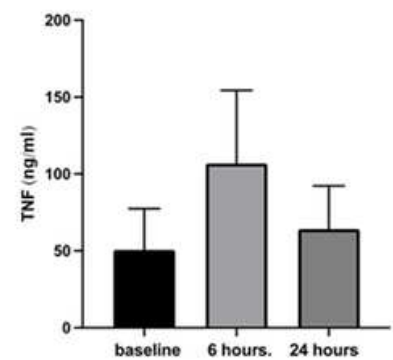

Figure 6

Inflammatory Response to Woodsmoke Particles of responsive volunteers as defined by \%PMNs at 24 hours The airway inflammatory response (cells and soluble phase mediators) to woodsmoke particles of 24-hour responders $(\mathrm{N}=28)$ and non-responders $(\mathrm{N}=13)$. 